Sir,

\section{Posterior lens nucleus displacement following intravitreal injection}

We report a case of posterior lens nucleus displacement following an intravitreal injection of bevacizumab (IVB). To the best of our knowledge this complication has not been previously reported.

\section{Case report}

A 67-year-old Caucasian male reported to the Eye casualty with complaints of persistent blurred vision in the left eye (OS) following IVB injection for retinal vein occlusion, 6 days earlier. The intravitreal injection had been performed from the infero-temporal quadrant. Best corrected visual acuity (BCVA) was hand movements, OS. Anterior segment examination was suggestive of cortical cataract and the state of the posterior capsule (PC) could not be identified (Figure 1). The ultrasonography B-scan was suggestive of a lens nucleus in the inferior vitreous cavity (Figure 2). BCVA prior to the injection was $6 / 36$.

A diagnosis of traumatic cataract with posterior nucleus displacement was made and therefore considered for vitreoretinal surgery. A dye-assisted anterior capsulorhexis was performed, only cortical matter in the capsular bag along with a large PC tear extending from 2 to 7 o'clock was noted. Following aspiration of cortical matter, the nucleus was removed via a pars-plana vitrectomy and lensectomy procedure. An intra-ocular lens (IOL) was placed in the sulcus. At 3 months follow-up, the BCVA was $6 / 24$ with a stable IOL and no vitreoretinal complications.

\section{Comment}

Although intravitreal injections are relatively safe procedures, there are reports of complications including vitreous prolapse, intraocular lens dislocation, and inadvertent capsule penetration. ${ }^{2,3,4}$ The injecting doctor mentioned that this patient had moved his eye during

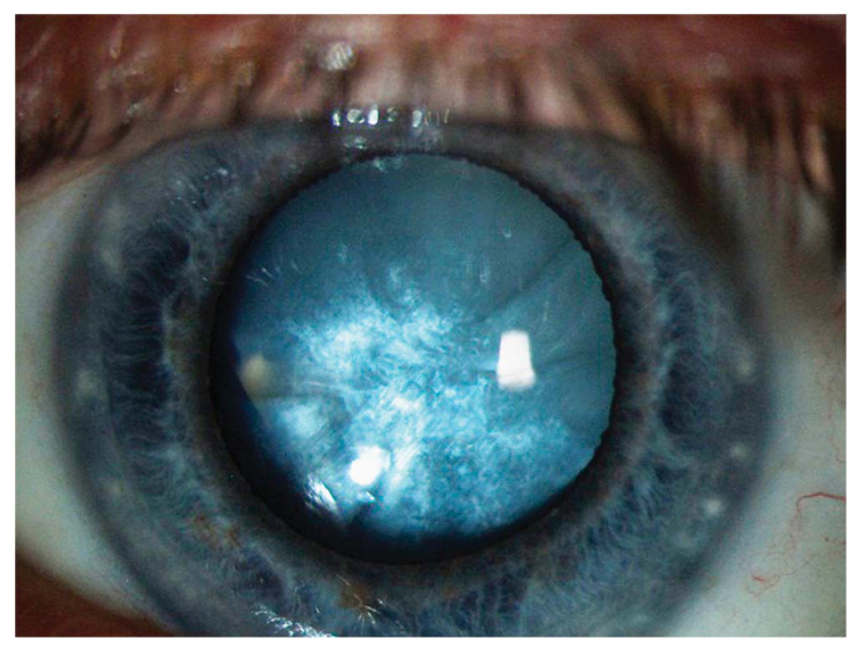

Figure 1 Slit-lamp photograph of the left eye obtained 6 days after the intravitreal injection, shows the presence of a traumatic cataract.

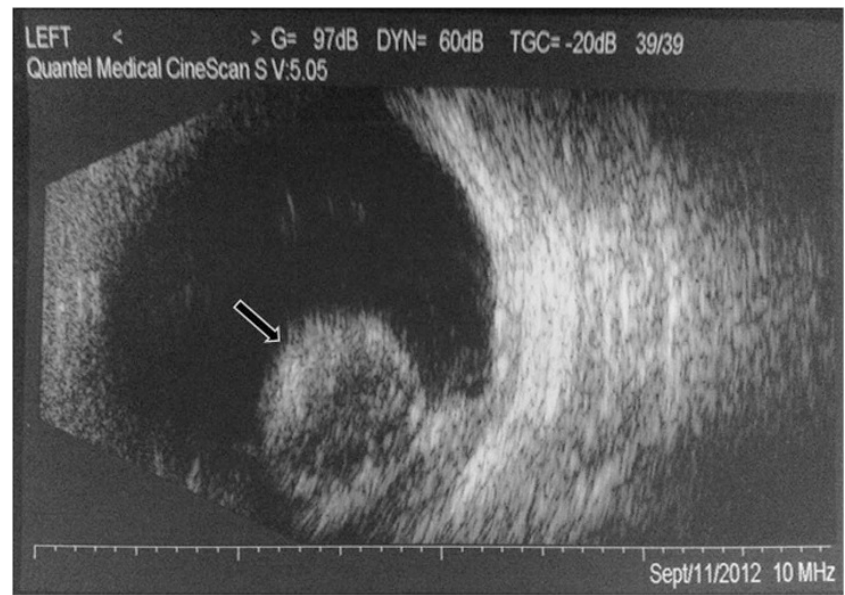

Figure 2 The ultrasonography B-scan image of the left eye shows the high-reflective, bi-convex, globular structure in the inferior vitreous cavity, suggestive of a posterior lens displacement (the arrow points to the lens). The retina was attached. 
the procedure and this possibly may have led to the posterior capsular damage by the 30 gauge needle. Our case reiterates the need for adherence to a few steps while performing the intravitreal injections. These include adequate warning to the patient immediately prior to the injection, maintaining correct direction of the needle at all times, that is, towards the mid vitreous cavity, right distance from limbus, and adequate anaesthesia. ${ }^{1,4,5}$ Indirect ophthalmoscopy should be performed at the end of the procedure.

To conclude, serious complications arising from a routine intravitreal injection can occasionally occur. It is important to adhere to meticulous injection techniques.

\section{Conflict of interest}

The authors declare no conflict of interest.

\section{References}

1 Frenkel RE, Haji SA, La M, Frenkel MP, Reyes A. A protocol for the retina surgeon's safe initial intravitreal injections. Clin Ophthalmol 2010; 4: 1279-1285.

2 Degenring RF, Sauder G. Vitreous prolapse and IOL dislocation during intravitreal injection of triamcinolone acetonide. Graefes Arch Clin Exp Ophthalmol 2006; 244(8): 1043-1044.

3 Saeed MU, Prasad S. Management of cataract caused by inadvertent capsule penetration during intravitreal injection of ranibizumab. J Cataract Refract Surg 2009; 35: 1857-1859.

4 Sampat KM, Garg SJ. Complications of intravitreal injections. Curr Opin Ophthalmol 2010; 21: 178-183.

5 Aiello LP, Brucker AJ, Chang S, Cunningham Jr, ET, D'Amico DJ, Flynn Jr, HW et al. Evolving guidelines for intravitreal injections. Retina 2004; 24: S3-S19.

V Felcida, S Habal and AK Tyagi

Department of Vitreoretinal Surgery, Birmingham \& Midland Eye Centre, Birmingham, UK

E-mail: vinayafelcida@yahoo.co.in

Eye (2013) 27, 1103-1104; doi:10.1038/eye.2013.140; published online 28 June 2013

\section{Sir,}

Are Do-It-Yourself companies setting a good example? A systematic study of ocular protection on the Internet

Do-It-Yourself (DIY) activities account for 30000 eye injuries annually in the United Kingdom. ${ }^{1}$ Despite this, the Royal Society of Prevention of Accidents ${ }^{2}$ found that $89 \%$ of Britons do not wear safety glasses or goggles when performing DIY activities associated with ocular morbidity such as hammering, drilling, and sawing. Desai $e \mathrm{al}^{3}$ conducted a 12-month retrospective study of all ocular injuries in Scotland and found that the single most common place for blinding traumatic injuries to occur was in the home $(52 \%)$.
We conducted a study to assess the promotion of eye protection on websites that advertise, educate, or promote the use of power tools. Ethical approval was not sought for the study.

A systematic search of 833 webpages in the power tools sections of the top 10 DIY company websites in the United Kingdom, identified using http:/ / www.google.co.uk, was conducted. Additionally, a search of 861 videos in the YouTube video channels of these websites was also conducted. Any headshot images of the power tool user were used for analysis in the study and the use of eye protection, with either safety glasses or goggles, was noted. Similarly, videos with clear headshot images of subjects using hammers, drills, or saws were also assessed.

A total of 44 webpages featured headshot images of users operating power tools. Promotional or instructional videos showing headshot footage were found in a total of 63 YouTube videos. On average, 39\% of website headshot images displayed models operating power tools without eye protection. For videos, an average of $59 \%$ of YouTube channel clips displayed models using hammers, drills, or saws without eye protection.

Therefore, our study has demonstrated that many DIY websites do not show adequate use of protective eyewear in photographs. Worryingly, out of three videos displaying DIY workshops for children, two videos displayed children using hammers and saws without eye protection.

We recommend that the findings of this study are considered by eye specialists, general practitioners, and by DIY companies, in the hope that better portrayal and promotion of ocular protection will occur.

\section{Conflict of interest}

The authors declare no conflict of interest.

\section{References}

1 The Royal Society of Prevention of Accidents. Home and Leisure Accident Surveillance System: 2000, 2001 and 2002 Data. Department of Trade and Industry. 2002.

2 College of Optometrists. DIY Eye Health Risk. http://www. college-optometrists.org/en/knowledge-centre/news/ index.cfm/DIY\%20Eye\%20Health\%20Risk. 2010. Accessed 2 February 2013.

3 Desai P, MacEwen CJ, Baines P, Minassian. DC. Incidence of cases of ocular trauma admitted to hospital and incidence of blinding outcome. Br J Ophthalmol 1996; 80: 592-596.

SR Rufai ${ }^{1}$, P Alexander ${ }^{1,2}$ and SC Lash ${ }^{2}$

${ }^{1}$ Faculty of Medicine, University of Southampton, Southampton, Hampshire, UK

${ }^{2}$ Department of Ophthalmology, University Hospital

Southampton, Southampton, Hampshire, UK

E-mail: sa1g09@soton.ac.uk

Eye (2013) 27, 1104; doi:10.1038/eye.2013.141;

published online 21 June 2013 